\title{
INVARIANT SUBSPACES OF TOEPLITZ OPERATORS WITH PIECEWISE CONTINUOUS SYMBOLS
}

\author{
VLADIMIR V. PELLER
}

(Communicated by Palle E. T. Jorgensen)

\begin{abstract}
Sufficient conditions are found for the existence of nontrivial invariant subspaces for Toeplitz operators with piecewise continuous symbols. The results are obtained by estimating the norm of the resolvents.
\end{abstract}

\section{INTRODUCTION}

This paper can be considered as a continuation of the papers [7, 8] where Toeplitz operators with continuous symbols were considered. Here we consider the same questions in the case of piecewise continuous symbols.

Recall that a subspace $L$ of a Banach space $X$ is called invariant (hyperinvariant) for an operator $T$ on $X$ if $T L \subset L \quad(R L \subset L$ for any operator $R$ commuting with $T)$. It is called nontrivial if $L \neq\{0\}$ and $L \neq X$. The investigation of invariant subspaces is one of the most important tools in operator theory (see [10]).

The question of the existence of a nontrivial invariant subspace for an arbitrary operator on Hilbert space (as well as for an arbitrary Toeplitz operator) remains open.

Given a bounded function $\varphi$ on the unit circle $\mathbf{T}$, the Toeplitz operator $T_{\varphi}$ on the Hardy class $H^{2}$ is defined by

$$
T_{\varphi} f=\mathbf{P}_{+} \varphi f, \quad f \in H^{2},
$$

where $\mathbf{P}_{+}$is the orthogonal projection from $L^{2}$ onto $H^{2}$. The function $\varphi$ is called the symbol of $T_{\varphi}$.

In [7] it was shown that if $\varphi \in C(\mathbf{T}), \varphi \neq$ const, the modulus of continuity $\omega_{\varphi}$ satisfies

$$
\int_{0} \frac{\omega_{\varphi}(t)}{t \log 1 / t} d t<\infty
$$

and there exists a Jordan Lipschitz arc $\Gamma$ in $\mathbf{C}$ such that $\varphi(\mathbf{T}) \cap J \cap \mathscr{O} \neq \varnothing$ and $\varphi(\mathbf{T}) \cap(\mathscr{O} \backslash J)=\varnothing$ for some open set $\mathscr{O}$, then $T_{\varphi}$ has a nontrivial hyperinvariant subspace.

Received by the editors January 21, 1992; the contents of this paper have been presented at the South East Annual Meeting of the American Mathematical Society, Charlotte, NC, 1991.

1991 Mathematics Subject Classification. Primary 47B35, 47A10, 47A15.

Key words and phrases. Toeplitz operator, modulus of continuity, resolvent. 
In [8] it was shown that if $\Gamma$ is a $C^{2}$-smooth curve, then (1) can be replaced by a weaker condition

$$
\int_{0} \frac{\omega_{\varphi}^{2}(t)}{t \log 1 / t} d t<\infty .
$$

It was also proved in [8] that (2) can be replaced by the condition

$$
\sum_{n \in \mathbf{Z}}|\widehat{\varphi}(n)|<\infty,
$$

where $\widehat{\varphi}(n)$ is the $n$th Fourier coefficient of $\varphi$. In [8] several other results on invariant subspaces of Toeplitz operators were obtained.

Note that earlier in $[3,4]$ the existence of nontrivial invariant subspaces was established under stronger assumptions on the symbol $\varphi$.

In this paper we find similar conditions in the case of Toeplitz operators with piecewise continuous symbols.

\section{Preliminaries}

2.1. Toeplitz operators. Let $P C$ denote the set of piecewise continuous functions on $\mathbf{T}$ that have finitely many jumps. For the sake of convenience we always assume that for $\varphi \in P C$

$$
\varphi(\zeta)=\varphi^{(-)}(\zeta), \quad \zeta \in \mathbf{T}
$$

where

$$
\varphi^{(+)}(\zeta) \stackrel{\text { def }}{=} \lim _{t \rightarrow 0-} \varphi\left(e^{i t} \zeta\right), \quad \varphi^{(-)}(\zeta) \stackrel{\text { def }}{=} \lim _{t \rightarrow 0+} \varphi\left(e^{i t} \zeta\right) .
$$

If $\varphi \in P C$ and $\varphi$ has a jump at $\zeta \in \mathbf{T}$, we denote by $I_{\zeta}$ the interval

$$
I_{\zeta}=\left[\varphi^{(-)}(\zeta), \varphi^{(+)}(\zeta)\right] .
$$

Then the essential spectrum

$$
\sigma_{e}\left(T_{\varphi}\right)=\left\{\lambda \in \mathbf{C}: T_{\varphi}-\lambda I \text { is not a Fredholm operator }\right\}
$$

admits the description

$$
\sigma_{e}\left(T_{\varphi}\right)=\varphi(\mathbf{T}) \cup \bigcup I_{\zeta},
$$

where the union is taken over all jumps $\zeta$ of $\varphi$.

Consider now the curve $\varphi^{\#}$ obtained from $\varphi$ by adjoining the intervals $I_{\zeta}$, where $\zeta$ ranges over the jumps of $\varphi$. Then for $\lambda \notin \sigma_{e}\left(T_{\varphi}\right)$ the operator $T_{\varphi}-\lambda I$ is invertible if and only if the winding number $\operatorname{wind}_{\lambda} \varphi^{\#}$ of $\varphi^{\#}$ with respect to $\lambda$ is zero.

For any $\lambda \notin \sigma_{e}\left(T_{\varphi}\right)$ we have

$$
\operatorname{ind}\left(T_{\varphi}-\lambda I\right)=-\operatorname{wind}_{\lambda} \varphi^{\#},
$$

where for a Fredholm operator $T$

$$
\text { ind } T \stackrel{\text { def }}{=} \operatorname{dim} \operatorname{Ker} T-\operatorname{dim} \operatorname{Ker} T^{*} \text {. }
$$

The same results also hold for piecewise continuous symbols, which can have infinitely many jumps (see [2]). We refer the reader to $[1,2,6,9,11]$ for more detailed information on Toeplitz operators. 
The above description of $\sigma\left(T_{\varphi}\right)$ can be reformulated in the following form. Let $\lambda \notin \varphi(\mathbf{T})$; then $T_{\varphi}-\lambda I$ is invertible if and only if $\varphi-\lambda$ admits a representation

$$
\varphi-\lambda=|\varphi-\lambda| \exp i \alpha,
$$

where $\alpha$ is a real-valued function in $P C$ such that

$$
\left|\alpha^{(-)}(\zeta)-\alpha^{(+)}(\zeta)\right|<\pi
$$

for any jump $\zeta$.

2.2. The Lyubich-Matsaev theorem. To prove the existence of nontrivial invariant subspaces we need the following result due to Lyubich and Matsaev [5].

Theorem. Let $T$ be an operator on a Banach space and $\Gamma$ a smooth arc on the plane. Assume that there is an open set $\mathscr{O}$ such that $\sigma(T) \cap \mathscr{O}=\Gamma \cap \mathscr{O} \neq \varnothing$. Let

$$
M(\delta) \stackrel{\text { def }}{=} \sup \left\{\left\|(\lambda I-T)^{-1}\right\|: \lambda \in \mathscr{O}, \operatorname{dist}(\lambda, \Gamma) \geq \delta\right\}
$$

If

$$
\int_{0} \log \log M(\delta) d \delta<\infty,
$$

then $T$ has a nontrivial hyperinvariant subspace.

2.3. Uniform polynomial approximation. In what follows we make use of the following theorem due to Jackson (see [12]).

Theorem. Let $\varphi \in C(\mathbf{T})$. Then

$$
\operatorname{dist}_{L^{\infty}}\left(\varphi, \mathscr{P}_{n}\right) \leq \operatorname{const} \omega_{\varphi}\left(\frac{\pi}{n+1}\right),
$$

where $\mathscr{P}_{n}$ is the set of trigonometric polynomials of degree at most $n$.

\section{EXISTENCE OF INVARIANT SUBSPACES}

In this section we obtain sufficient conditions for a Toeplitz operator with piecewise continuous symbol to have a nontrivial hyperinvariant subspace.

Let $\varphi$ be a function in $P C$. We define the modulus of continuity $\omega_{\varphi}$ as follows. Let $\xi_{1}, \ldots, \xi_{m}$ be the jumps of $f$ and $\Gamma_{1}, \ldots, \Gamma_{m}$ the complementing arcs to $\xi_{1}, \ldots, \xi_{m}$. Then $f$ is continuous on each $\Gamma_{j}, j=1,2, \ldots, m$, and the modulus of continuity $\omega_{\varphi}$ is defined by

$$
\omega_{\varphi}(\delta)=\max _{1 \leq j \leq m} \omega_{\varphi \mid \Gamma_{j}}(\delta)
$$

where

$$
\omega_{\varphi \mid \Gamma_{j}}=\sup \left\{|\varphi(\zeta)-\varphi(\tau)|: \zeta, \tau \in \Gamma_{j},|\zeta-\tau| \leq \delta\right\} .
$$

Recall that given $\varphi \in P C$, to each jump $\xi$ of $\varphi$ there corresponds the interval $I_{\xi}$ defined by (3).

The following theorems are the main results of the paper. 
Theorem 1. Let $\varphi \in P C$ and $\zeta$ be a jump of $\varphi$. Suppose that $I_{\zeta} \not \subset \varphi(\mathbf{T})$ and

$$
\int_{0} \frac{\omega_{\varphi}(t)}{t \log 1 / t} d t<\infty .
$$

Then $T_{\varphi}$ has a nontrivial hyperinvariant subspace.

Theorem 2. Let $\varphi \in P C$ and $\zeta$ be a jump of $\varphi$. Suppose that there is an open set $\mathscr{O}$ such that

$$
\mathscr{O} \cap I_{\zeta} \neq \varnothing \quad \text { and } \varphi(\mathbf{T}) \cap\left(\mathscr{O} \backslash I_{\zeta}\right)=\varnothing
$$

Suppose also that

$$
\int_{0} \frac{\left(\omega_{\varphi}(t)\right)^{1 / 2}}{t \log 1 / t} d t<\infty .
$$

Then $T_{\varphi}$ has a nontrivial hyperinvariant subspace.

To prove the above theorems we need a quantitative version of the DevinatzWidom theorem (see [1, 11]). That theorem claims that a Toeplitz operator $T_{u}$ with a unimodular symbol $u$ (i.e., $|u(\zeta)|=1$ for almost all $\zeta$ in $\mathbf{T}$ ) is invertible if and only if $u$ can be represented in the form

$$
u=\exp i(\alpha+\widetilde{\beta}+c)
$$

where $\alpha$ and $\beta$ are bounded real-valued functions, $c \in \mathbf{R},\|\alpha\|_{\infty}<\pi / 2$, and $\widetilde{\beta}$ is the harmonic conjugate of $\beta$ (see [13]). We need the following estimate for $\left\|T_{u}^{-1}\right\|$.

Lemma 1. Let $u$ be a function defined by (6), where $\alpha, \beta \in \operatorname{Re} L^{\infty}, c \in \mathbf{R}$, $\|\alpha\|_{\infty}<\pi / 2$. Then

$$
\left\|T_{u}^{-1}\right\| \leq \text { const } e^{\|\beta\|_{\infty}} / \rho
$$

where $\rho=\pi / 2-\|\alpha\|_{\infty}$.

Proof. Clearly, without loss of generality we can assume that $c=0$. Put

$$
h=\exp \frac{1}{2}(\beta+i \widetilde{\beta}) \text {. }
$$

Then $h$ is an outer function invertible in $H^{\infty}$ and $T_{u}=T_{1 / h} T_{e^{i n}} T_{h}$. So $T_{u}^{-1}=$ $T_{1 / h} T_{e^{i o}}^{-1} T_{\bar{h}}$, which implies

$$
\left\|T_{u}^{-1}\right\| \leq\|1 / h\|_{\infty}\|h\|_{\infty}\left\|T_{e^{i n}}^{-1}\right\|=e^{\|\beta\|_{x}}\left\|T_{e^{\prime \prime \prime}}^{-1}\right\| .
$$

It remains to estimate $\left\|T_{e^{\text {er }}}^{-1}\right\|$. We have

$$
\text { Range } e^{i \alpha} \subset\left\{e^{i \vartheta}:-\pi / 2+\rho \leq \vartheta \leq \pi / 2-\rho\right\} \text {. }
$$

Put $\varepsilon=\sin \rho$. Then

$$
\text { Range } \varepsilon e^{i \alpha} \subset\left\{\varepsilon e^{i \vartheta}:-\pi / 2+\rho \leq \vartheta \leq \pi / 2-\rho\right\} .
$$

It is easy to see that

$$
\left\|1-\varepsilon e^{i \alpha}\right\|_{\infty}=\left|1-\varepsilon e^{i(\pi / 2-\rho)}\right|=\cos \rho .
$$

We have $T_{\varepsilon \exp (i \alpha)}=I-T_{1-\varepsilon \exp (i \alpha)}$ and $\left\|T_{1-\varepsilon \exp (i \alpha)}\right\|=\cos \rho<1$. Therefore

$$
\left\|T_{\varepsilon \exp (i \alpha)}\right\| \leq \frac{1}{1-\cos \rho}
$$


which implies

$$
\left\|T_{\exp (i \alpha)}^{-1}\right\| \leq \frac{\sin \rho}{1-\cos \rho} \leq \text { const } \frac{1}{\rho} .
$$

In the proof of the above theorems, an important role will be played by the function $\nu$ defined on $\left[0, \sup _{\delta>0} \omega_{\varphi}(\delta)\right]$ by

$$
\nu(s)=\sup \left\{t: \omega_{\varphi}(t) \leq s\right\}
$$

Proof of Theorem 1. Let $\mathscr{O}$ be an open set such that

$$
\mathscr{O} \cap I_{\zeta} \neq \varnothing, \quad \mathscr{O} \cap \varphi(\mathbf{T})=\varnothing,
$$

and $\mathscr{O} \backslash I_{\zeta}$ is disjoint with $I_{\xi}$ for any jump $\xi$.

Consider first the case when $\sigma_{e}\left(T_{\varphi}\right) \neq \sigma\left(T_{\varphi}\right)$. Let $\lambda \in \sigma\left(T_{\varphi}\right) \backslash \sigma_{e}\left(T_{\varphi}\right)$. Then either $\operatorname{Ker}\left(T_{\varphi}-\lambda I\right) \neq\{\boldsymbol{0}\}$ or $\operatorname{Ker}\left(T_{\varphi}-\lambda I^{*}\right) \neq\{\boldsymbol{0}\}$ and both $\operatorname{Ker}\left(T_{\varphi}-\lambda I\right)$ and $\left(\operatorname{Ker}\left(T_{\varphi}-\lambda I\right)^{*}\right)^{\perp}$ are hyperinvariant subspaces of $T_{\varphi}$, one of which is nontrivial.

Suppose now that $\sigma_{e}\left(T_{\varphi}\right)=\sigma\left(T_{\varphi}\right)$. In this case $T_{\varphi}-\lambda I$ is invertible for any $\lambda \in \mathscr{O} \backslash I_{\zeta}$ (see $\left.\S 2.2\right)$. Let us estimate the resolvent $\left(T_{\varphi}-\lambda I\right)^{-1}$.

It follows from the invertibility of $T_{\varphi}-\lambda I$ that $\varphi-\lambda$ admits a representation

$$
\varphi-\lambda=|\varphi-\lambda| \exp i v_{\lambda}
$$

where $v_{\lambda} \in P C, v_{\lambda}$ has jumps at the jumps of $\varphi$, and $\left|v_{\lambda}^{(+)}(\xi)-v_{\lambda}^{(-)}(\xi)\right|<\pi$ for any jump $\xi$ of $\varphi($ see $\S 2.2)$.

It is easy to see that such $v_{\lambda}$ can be represented as

$$
v_{\lambda}=f_{\lambda}+g_{\lambda},
$$

where $f_{\lambda} \in C(\mathbf{T}), g_{\lambda} \in P C,\left\|g_{\lambda}\right\|_{\infty} \leq \pi / 2$.

Moreover, it is also clear that for $\lambda \in \mathscr{O} \backslash I_{\zeta}$ the modulus of continuity $\omega_{f_{i}}$ admits the estimate

$$
\omega_{f_{\lambda}}(\delta) \leq \text { const } \omega_{\varphi}(\delta) .
$$

Note also that $\left|g_{\lambda}^{(+)}(\xi)-g_{\lambda}^{(-)}(\xi)\right|$ is the angle at which the interval $I_{\xi}$ is seen from the point $\lambda$.

We have

$$
\varphi-\lambda=\bar{h}_{\lambda} \exp i\left(f_{\lambda}+g_{\lambda}\right) h_{\lambda}
$$

where $h_{\lambda}$ is an outer function such that $\left|h_{\lambda}\right|^{2}=|\varphi-\lambda|$. We have

$$
\left\|T_{\varphi-\lambda}^{-1}\right\| \leq\left\|T_{1 / h_{\lambda}}\right\| \cdot\left\|T_{1 / \bar{h}_{\lambda}}\right\| \cdot\left\|T_{\exp i\left(f_{\lambda}+g_{\lambda}\right)}^{-1}\right\| .
$$

Obviously

$$
\left\|T_{1 / h_{\lambda}}\right\| \cdot\left\|T_{1 / \bar{h}_{\lambda}}\right\|=\|1 /|\varphi-\lambda|\|_{\infty} \leq \text { const } 1 / \delta,
$$

where $\delta \stackrel{\text { def }}{=} \operatorname{dist}\left(\lambda, I_{\zeta}\right)$.

Let us estimate $\left\|T_{\exp i\left(f_{\lambda}+g_{\lambda}\right)}^{-1}\right\|$. Clearly

$$
\pi / 2-\left\|g_{\lambda}\right\|_{\infty} \geq c \delta, \quad \lambda \in \mathscr{O} \backslash I_{\zeta},
$$

for some constant $c$. 
By Jackson's theorem (see $§ 2.3$ )

$$
\operatorname{dist}_{L^{\infty}}\left(f_{\lambda}, \mathscr{P}_{n}\right) \leq \text { const } \omega_{f_{\lambda}}\left(\frac{\pi}{n+1}\right) \leq d \omega_{\varphi}\left(\frac{\pi}{n+1}\right)
$$

for some constant $d$. Let $n_{\lambda}$ be the smallest integer for which

$$
d \omega_{\varphi}\left(\frac{\pi}{n_{\lambda}+1}\right) \leq \frac{c}{2} \delta .
$$

By the definition of $\nu$ we have

$$
n_{\lambda} \leq \frac{\pi}{\nu(c \delta / 2 d)}
$$

So there exists a polynomial $P_{\lambda}$ in $\mathscr{P}_{n_{\lambda}}$ such that

$$
\left\|f_{\lambda}-P_{\lambda}\right\|_{\infty} \leq \frac{c \delta}{2}
$$

Obviously $\left\|P_{\lambda}\right\|_{\infty} \leq$ const $\cdot\left\|f_{\lambda}\right\|_{\infty} \leq$ const .

Let $Q_{\lambda}=-\widetilde{P}_{\lambda}$. Then $Q_{\lambda} \in \mathscr{P}_{n_{\lambda}}$ and $\widetilde{Q}_{\lambda}=P_{\lambda}-\widehat{P}_{\lambda}(0)$. We have

$$
f_{\lambda}+g_{\lambda}=\alpha+\tilde{\beta}+c
$$

where $\alpha=g_{\lambda}+f-P_{\lambda}, \beta=-Q_{\lambda}, c=\widehat{P}_{\lambda}(0)$. It follows from (14) and (16) that

We can now apply Lemma 1 and obtain

$$
\frac{\pi}{2}-\|\alpha\|_{\infty} \leq \frac{c \delta}{2}
$$

$$
\left\|T_{\exp i\left(f_{\lambda}+g_{\lambda}\right)}^{-1}\right\| \leq \frac{\text { const }}{\delta} \exp \left\|Q_{\lambda}\right\|_{\infty}
$$

It is well known (see [13]) that

$$
\left\|Q_{\lambda}\right\|_{\infty}=\left\|\widetilde{P}_{\lambda}\right\|_{\infty} \leq \text { const } \log n_{\lambda}
$$

Thus it follows from (12), (13), and (17) that

$$
\left\|T_{\varphi-\lambda}^{-1}\right\| \leq \frac{\text { const }}{\delta^{2}} n_{\lambda}
$$

which together with (15) yields

$$
\left\|T_{\varphi-\lambda}^{-1}\right\| \leq \frac{\text { const }}{\delta^{2}} \frac{1}{\nu(c \delta / 2 d)} .
$$

Now it is easy to see that the hypotheses of the Lyubich-Matsaev theorem (with $\Gamma=I_{\zeta} \cap \mathscr{O}$ ) will be satisfied if we show that

$$
\int_{0} \log \log \frac{1}{\nu(\delta)} d \delta<\infty
$$

But this follows from the following lemma proved in [7].

Lemma 2. Inequalities (4) and (18) are equivalent to each other.

Let us proceed to the proof of Theorem 2.

Proof of Theorem 2. The proof is analogous to that of Theorem 1. As in Theorem 1 we can assume that $\sigma_{e}\left(T_{\varphi}\right)=\sigma\left(T_{\varphi}\right)$ and $\mathscr{O} \backslash I_{\zeta}$ is disjoint with $I_{\xi}$ for 
any jump $\xi$. Then every $\lambda$ in $\mathscr{O} \backslash I_{\zeta}$ is in the resolvent set and we can obtain the factorization (8), the representation (9), the factorization (11), and the estimates (12) and (13).

The only problem that can arise in this case is that inequality (10) can fail under the hypotheses of Theorem 2. Indeed, $\varphi$ is allowed now to take values in $I_{\zeta} \cap \mathscr{O}$, which can lead to a distortion of the modulus of continuity of $f_{\lambda}$ as $\lambda$ tends to $I_{\zeta}$. However it is easy to see that in this case the following estimate holds:

$$
\omega_{f_{\lambda}}(\delta) \leq \frac{\text { const }}{\delta} \omega_{\varphi}(\delta)
$$

So by Jackson's theorem (see $\S 2.3$ ) we can find a polynomial $P_{\lambda}$ of degree $n_{\lambda}$ such that

$$
\left\|f_{\lambda}-P_{\lambda}\right\|_{\infty} \leq \text { const } \omega_{f_{\lambda}}\left(\frac{\pi}{n+1}\right) \leq \frac{d}{\delta} \omega_{\varphi}\left(\frac{\pi}{n+1}\right)
$$

for some constant $d$. It follows that $n_{\lambda}$ can be chosen as the smallest integer for which

$$
d \omega_{\varphi}\left(\frac{\pi}{n_{\lambda}+1}\right) \leq \frac{c}{2} \delta
$$

which is equivalent to $n_{\lambda} \leq \pi / \nu\left(c \delta^{2} / 2 d\right)$.

Then the proof repeats the arguments used in the proof of Theorem 1, which leads to the estimate

$$
\left\|T_{\varphi-\lambda}^{-1}\right\| \leq \frac{\text { const }}{\delta^{2}} \frac{1}{\nu\left(c \delta^{2} / 2 d\right)} .
$$

So the hypotheses of the Lyubich-Matsaev theorem will be satisfied if we show that

$$
\int_{0} \log \log \frac{1}{\nu\left(\delta^{2}\right)} d \delta<\infty
$$

which is equivalent to (5) by the following lemma.

Lemma 3. Inequalities (5) and (19) are equivalent to each other. [7]).

The proof of Lemma 3 is completely analogous to the proof of Lemma 2 (see

\section{REFERENCES}

1. R. G. Douglas, Banach algebra techniques in operator theory, Academic Press, New York, 1972.

2. Banach algebra techniques in the theory of Toeplitz operators, CBMS Regional Conf. Ser. in Math., vol. 15, Amer. Math. Soc., Providence, RI, 1973.

3. E. M. Dyn'kin, Theorems of Wiener-Lévy type and estimates for Wiener-Hopf operators, Mat. Issled. 8 (1973), 14-25. (Russian)

4. L. C. Gambler, A study of rational Toeplitz operators, Ph.D. Thesis, SUNY at Stony Brook, NY, 1977.

5. Yu. I. Lyubich and V. I. Matsaev, Operators with separable spectrum, Mat. Sb. 56 (98) (1962), 433-468; English transl. in Amer. Math. Soc. Transl. (2) 47 (1965).

6. N. K. Nikol'skii, Treatise on the shift operator, Springer-Verlag, Berlin and New York, 1986.

7. V. V. Peller, Invariant subspaces of Toeplitz operators, Zap. Nauchn. Sen Leningrad. Otdel. Mat. Inst. Steklov. (LOMI) 126 (1983), 170-179; English transl. in J. Soviet Math. 27 (1984). 
8. __ Spectrum, similarity, and invariant subspaces of Toeplitz operators, Izv. Akad. Nauk SSSR Ser. Mat. 50 (1986), 776-787; English transl., Math. USSR-Izv. 29 (1987), 133-144.

9. V. V. Peller and S. V. Khrushchev, Hankel operators, best approximations, and stationary Gaussian processes, Uspekhi Mat. Nauk 37 (1982), no. 1, 53-124; English transl. in Russian Math. Surveys 37 (1982).

10. H. Radjavi and P. Rosenthal, Invariant subspaces, Springer-Verlag, Berlin and New York, 1973.

11. D. Sarason, Function theory on the unit circle, Lecture Notes, Dept. Math., Virginia Polytechnic Inst. and State Univ., Blacksburg, VA, 1978.

12. A. F. Timan, Theory of approximation of functions of a real variable, Fizmatgiz, Moscow, 1960; English transl., Pergamon Press, Oxford, and Macmillan, New York, 1963.

13. A. Zygmund, Trigonometric series, 2nd rev. ed., Vols. I, II, Cambridge Univ. Press, London and New York, 1959.

Department of Mathematics, University of Lancaster, Lancaster, LA1 4YL United KINGDOM

St.-Petersburg Branch, Steklov Institute of Mathematics, Fontanka 27, 191011 St.-Petersburg, Russia

Current address: Department of Mathematics, Texas A \& M University, College Station, Texas 77843

E-mail address: peller@math.tamu.edu 